\title{
Absence of Nocturnal Penile Tumescence: An Earlier Sign of Erectile Dysfunction and Its Linked Comorbid Conditions
}

\author{
Inder MS Sandhu' ${ }^{1}$, Neeti R Singh ${ }^{2}$
}

\begin{abstract}
Most of the patients with vascular and metabolic disorders such as cardiovascular diseases (CVDs), hypertension, diabetes, obesity, or stroke show some kind of abnormal erectile function (EF) significantly affecting the quality of life (QoL). There is a direct link between person's sexual health and physical health. Nocturnal penile tumescence (NPT), the involuntary erections occur during sleep, are the normal physiological and spontaneous phenomenon in all healthy men. Nocturnal penile tumescence measurement expedites the procedure of differentiating organic causes of erectile dysfunction (ED) in men from psychological causes. Absence of NPT can precede ED and its related comorbid conditions and should be given importance during early clinical diagnoses of such disorders.
\end{abstract}

Keywords: Comorbid conditions, Erectile dysfunction, Nocturnal penile tumescence, Vascular and metabolic disorders.

AMEl's Current Trends in Diagnosis \& Treatment (2019): 10.5005/jp-journals-10055-0074

\section{INTRODUCTION}

Considering the amount of data demonstrating a strong correlation between nocturnal penile tumescence (NPT), erectile dysfunction (ED), and several life-risky comorbid conditions like hypertensive disorders, hyperlipidemia, diabetes mellitus, diseases of cardiovascular system, state of corpulence, dysthymia, and chronic obstructive pulmonary disease, ${ }^{1-5}$ a conscientious and extensive general health assessment of patients complaining of NPT or ED should be carried out. A physician can enquire about early signs of the abovesaid comorbid conditions while dealing a patient with NPT and ED or the vice versa. Awareness among public in general and clinicians in particular about using NPT as an earlier marker for many life-threatening conditions can help in reducing mortality rates marginally in such cases. ${ }^{6}$

\section{Sexual Health}

World Health Organization defines sexual health as "a state of complete physical, mental and social wellbeing and not merely the absence of disease or infirmity, in all matters relating to the reproductive system and to its functions and processes". ${ }^{7}$ In men, sexual dysfunction includes ED, failure of the gonads, testosterone deficiency, and ejaculatory disorders, primarily the premature ejaculation. These conditions could be highlighted as life-threatening issues because of their direct association with other severe vascular and metabolic disorders and not just the quality of life (QoL) index. Despite there is availability of effective treatment of sexual dysfunctions, a significant number of men affected by these conditions are not getting appurtenant treatment. ${ }^{6}$ Numerous studies have clearly established a direct link between person's sexual health and physical health. There is a straight forward relationship between erectile function (EF) and hypertension, dyslipidemia, obesity, diabetes, and other cardiovascular risk factors. ${ }^{1-3,8,9}$ In last few decades, measurement of NPT was developed justly to assess the EF and plays a major role in the diagnosis of ED as these measurements aid in differentiating organic causes of ED from psychogenic causes. ${ }^{4}$
1Department of Obstetrics and Gynaecology, Sri Guru Ram Das Institute of Medical Sciences and Research, Amritsar, Punjab, India

${ }^{2}$ Department of General Surgery, Sri Guru Ram Das Institute of Medical Sciences and Research, Amritsar, Punjab, India

Corresponding Author: Inder MS Sandhu, Department of Obstetrics and Gynaecology, Sri Guru Ram Das Institute of Medical Sciences and Research, Amritsar, Punjab, India, Phone: +91 0180 2870200, e-mail: ims_sandhu@yahoo.com

How to cite this article: Sandhu IMS, Singh NR. Absence of Nocturnal Penile Tumescence: An Earlier Sign of Erectile Dysfunction and Its Linked Comorbid Conditions. AMEl's Curr Trends Diagn Treat 2019;3(2):72-76.

Source of support: Nil

Conflict of interest: None

\section{Erectile Dysfunction}

Erectile dysfunction can be defined as "the persistent or recurrent inability to attain and maintain an erection sufficient to perform sexual activity". ${ }^{10}$ Erectile function is a phenomenon that involves psychoneurovascular functions which finally end up with an increase in arterial flow of blood within the hypogastric-penile bed, which in turn results in the activation of the veno-occlusive system of the corpora cavernosa. Erectile dysfunction is generally categorized as organic, psychogenic, or a combination of both. ${ }^{11,12}$ Young adults make major part of patients of psychogenic ED (pED). The pED contributes around $85.2 \%$ of patients of ED who are under the age of 40 years, whereas the incidence decreases to $40 \%$ if the patients are older than 40 years. There is a link between increased prevalence of ED with increasing age. ${ }^{13,14}$ The ED can be a result of many conditions such as hormonal, drug induced, neurogenic, anatomic, vasculogenic, or psychogenic. ${ }^{15,16}$ These conditions can be present in any individual and may be manifested as hypertensive disorders, hyperlipidemia, diabetes mellitus, diseases of cardiovascular system, state of corpulence, dysthymia, chronic obstructive pulmonary disease, benign prostatic hypertrophy, or lower urinary tract symptoms. ${ }^{9,13,17,18}$ The most prevalent risk factors 
that are found in atherosclerosis have also been frequently present in patients with ED. Erectile dysfunction in most of the cases is the clinical manifestation of abnormal functioning of vascular system affecting penile circulation as a part of a more generalized vascular disorder. ${ }^{19}$ Detailed medical history and hormone analysis are the basic prerequisites for the diagnosis of ED. There should be clinical practice to differentiate between organic ED and pED and many international guidelines are already available to guide clinicians in this regard. ${ }^{20}$

\section{Erectile Dysfunction and Stroke}

Prevalence of ED is very high in patients following a stroke. Diabetes, hypercholesterolemia, and hypertension are more common conditions that are present among stroke patients with ED than in stroke patients who are without ED. ${ }^{14}$ After stroke, sexual activity, function, and satisfaction level often changes. ${ }^{21,22}$ Most of the sexual activity domains, such as intercourse satisfaction, sexual desire, orgasmic function, and most important the EF, show lower scores in all stroke patients with ED. ${ }^{14}$ In many cases, premorbid medical conditions such as hypertension, cardiac diseases, and diabetes can affect the savor of sexual life in stroke patients. ${ }^{23}$ Many studies have shown that stroke patients experience sexual dysfunction and feel lament toward sexual activities after stroke. ${ }^{21,24,25} \mathrm{~A}$ study by Ponholzer et al. has shown that men with moderate or severe ED were at $43 \%$ higher risk of a stroke within 10 years. This increased risk was present in all age-groups but more significant in higher age groups. ${ }^{26}$ Jung et al. in their study of 109 stroke patients have shown that the constancy of intercourse and libido decreased after stroke along with severe ED. Erectile function significantly gets decreased in patients with multiple brain lesions as compared with the patients with one lesion. As sexual desire and ejaculatory disorders might have an association with left basal ganglia and the right cerebellum, respectively, the specific lesions in these locations during stroke show significant link between stroke and these disorders. ${ }^{27}$

\section{Erectile Dysfunction and CVD}

Cardiovascular disease (CVD) is apparently the biggest prevailing concurrent condition in men with ED. ${ }^{17}$ Men with the ED condition are more prone to coronary heart disease or stroke as compared to men without this condition, ${ }^{26,28}$ these conditions are also linked conversely. ${ }^{29}$ Cardiovascular disease and ED portage undistinguished risk factors, because endothelial dysfunction is the shared underlying factor in both conditions. An intact vascular endothelium is required for a normal EF as it is a neurovascular phenomenon, and this may be the reason that factors which affects the arterial vasculature might also disturb EF adversely. ${ }^{30}$ Even in case of absence of CVD in many males with ED, it has been seen that they have very few bone marrow-derived endothelial progenitor cells which are required to promote endothelial repair, which is an evidence indicator that ED can be seen as the first and foremost clinical sign of peripheral vascular disease. ${ }^{31,32}$ This is the reason that vascular risk factors such as hyperlipidemia, diabetes, hypertension, and atherosclerosis are directly linked to the severity level of ED. ${ }^{33,34}$ Erectile dysfunction can be a more appropriate medium to predict the resulting cardiovascular events than microalbuminuria, hypertension, glycated hemoglobin A1, or lipid panel. ${ }^{17}$ Atherosclerosis and endothelial dysfunction, which in turn give rise to disorders of coronary and penile circulation, may be an end product of interaction between androgens, chronic inflammation, and various cardiovascular risk factors, and might be the predicament which shows the link between CVD and ED. As the size of penile artery is tinier in comparison to coronary arteries, when the endothelial dysfunction level is same, it accounts for a more compelling attrition of blood flow in erectile tissues in comparison to the coronary blood transmission. This suggests that ED could be an important prior clue of systemic endothelial dysfunction. From a clinical point of view, as CVD may follow ED, so ED can act as a preceding signal to spot men at greater risk of CVDs. As ED patients are at elevated risk of developing CVD, they should go through comprehensive cardiovascular evaluation and should be given an accelerated treatment of all risk factors. ${ }^{35}$

\section{Erectile Dysfunction-Obesity and Testosterone}

All three causes of ED that is physical illness (organic component), stress (intrapsychic component), and relationship difficulties (relationship component), are all affected by testosterone levels. Most important function of sexual desire is the timing of erectile process and coordination of penile erection during sex, which is the main physiological action in the course of male sexual response and is regulated by testosterone. ${ }^{36}$ The processes of penile erection and regulation of male sexual behavior and attitudes are controlled by the levels of testosterone in blood. The conditions such as diabetes, obesity, insulin resistance, and other metabolic syndromes are significantly linked with low levels of testosterone. ${ }^{37}$ Excess fat accumulation could be a result of low testosterone levels in body. Despite the fact that lean patients are mostly less insulin resistant than obese ones, the state of being obese is not always linked to insulin resistance. ${ }^{38}$ Many scientific studies have brought to light that obesity have a correlation with progression and prevalence of $E D$, though high body mass index (BMI) in itself may not be the only cause of ED. But it may be the case that the predisposition of a patient to vascular and metabolic impairments which are caused by high BMI in turn leads to ED. ${ }^{39-41}$ Regulation of male sexual function gets disturbed by high body mass and insulin resistance as these can lead to decrease in free and total testosterone levels. ${ }^{42}$ Hypogonadism could be studied as one of the diverse adverse fallouts of being hefty and obese. In a study by Bacon et al., BMI of more than 28.7 showed a 30\% higher link to ED than a BMI of less than 25 in men. ${ }^{43}$ In another study by Chung et al., it has been found that obese men with ED have higher incidence of hypercholesterolemia, diabetes, and other risk factors associated with CVDs than nonobese men with ED. ${ }^{44}$ Furthermore, in another study, the levels of sex hormone binding globulin (SHBG)-bound and unbound testosterone decline in males who are obese in ratio to the levels of their obesity. ${ }^{45}$ To support this, data from highly obese men have shown that the levels of luteinizing hormone and pulse amplitude modulations were constricted in comparison to controls with normal weight. These studies back the concept of hypoandrogenism which is purely obesity associated. ${ }^{46,47}$ Another study also showed that if men remain in obese state for around 10 years or more, they have a greater chance of decrease in free and total testosterone levels; and by treating their low testosterone levels, their ED condition improves. ${ }^{48}$ Improvement in sexual function, cardiovascular profile, and higher circulating levels of testosterone can be achieved by simple lifestyle modifications, which can lead to weight loss or decrease in central obesity. ${ }^{49,50}$ Few studies exhibit that visceral fat disposition can be affected by the androgenic milieu. This can be done by using testosterone replacement therapy 
in subjects with condition of hypogonadism, which in process helps in decreasing the fat mass, resulting in better lipid profile and improved sexual dysfunction. ${ }^{51-53}$ In fact, hypogonadism can be considered as a direct affect of visceral obesity and may be a direct cause or due to increased insulin resistance and vice versa can also the case be, which in turn establishes a vicious cycle. ${ }^{42}$ Several markers of inflammation like proinflammatory cytokines show the increased levels in obese persons. These markers have correlation with endothelial dysfunction which can be exhibited by reduced platelet aggregation, decreased blood pressure, or increased levels of markers of low-grade inflammation. ${ }^{53}$

\section{Erectile Dysfunction, Diabetes, HyperLipidemia, and Hypertension}

The association of diabetes mellitus, low level of testosterone, hypogonadism, and other metabolic syndrome with ED is a wellknown fact due to the high prevalence of ED in these patients. Conditions, such as hypertension, hyperglycemia, high cholesterol, obesity especially around abdomen, hypertriglyceridemia, low high-density lipoprotein, help in specifically identifying individuals who are at high risk of metabolic diseases and CVDs and can also be used as early diagnostic tools for detecting ED..$^{17,54,55}$ Men with diabetes have 3 to 4 times more chance of developing ED compared to men without diabetes. The chances of hypogonadism is also double for such males and that is the reason why ED is much more prevalent, severe, and permanent in men with diabetes in comparison to the normal population. ${ }^{56,57}$ When both diabetes mellitus and hypogonadism are present in an individual, it can exasperate the condition of sexual dysfunction as penile vascular reactivity can further get compromised. Level of cholesterol and low-density lipoprotein (LDL) are found to be higher in patients with ED. The LDL is the main bearer of cholesterol in blood, and it plays major role in development of coronary atherosclerosis by inhibiting the endothelium-dependent relaxation of cavernous smooth muscles. By treating hyperlipidemia, the ED condition can be improved..$^{13}$ It has been reported that in some studies ED is more common among persons with hypertension as compared to matched healthy controls. ${ }^{58}$ Another study by Dusing $R$ found that when hypertensive patients were treated with valsartan therapy the degree of ED decreased exponentially. ${ }^{59}$ As prevalence of diabetes, hypertension, melancholy, and hyperlipidemia is found to be very high, it supports the hypothesis that these conditions share common risk factors with ED. The theory which claims that the clinicians should include ED in the evaluation profiling for the abovesaid conditions gets more weightage and thus ED could be used as a possible observable marker for these diseases. ${ }^{60}$

\section{Nocturnal Penile Tumescence}

Nocturnal penile tumescence refers to erections that occur during sleep in males. These erections are very much normal and happen almost every night throughout a man's life, but without any sexual stimulation. Also known as "Morning wood", NPT is an unprompted firmness of the phallus while at sleep or at the time of waking up. Nocturnal penile tumescence is present in all men who have no physiological ED; normally three to five times in the course of night's sleep. Rapid eye movement sleep time is the peak of this phenomenon. ${ }^{61,62}$ Nocturnal penile tumescence is supposed to promote penile health. Nocturnal penile tumescence helps to regulate oxygen-dependent biological processes through which it helps to preserve the morphological and dynamic coherence of corpora cavernosa; this is the reason that all healthy men all through their life experience NPT. ${ }^{63}$ Neurovascular and spinal mechanisms and hormonal control are of great significance in nocturnal erectile activity. Normal NPT readings or NPT and rigidity (NPTR) indices are good signs of intactness of penile structure as well as neural and vascular supplies to penis. ${ }^{64}$ However, NPT decreases with increasing age. ${ }^{65}$ Assessment of EF using NPT helps in the diagnosis of ED and facilitates in distinguishing organic causes from psychological causes ${ }^{4}$ and suggests that the patient has $p E D$ rather than neurogenic or vascular ED. To distinguish $p E D$ from organic ED, the monitoring of NPTR with RigiScan is one of the most authentic techniques. Rigiscan readings in case of $p E D$ will be in normal range, but these readings with abnormal range will be an indicator showing presence of organic ED. Central nervous system is also involved in pED which was exhibited successively with the help of functional and anatomical magnetic resonance imaging. ${ }^{5}$ Nocturnal penile tumescence or sleep-related erections and their link with rapid eye movement was found long ago in scientific literature. ${ }^{66}$ Predominantly seven techniques or methods are available, with some modifications, which are mainly used to find NPTR. These methods include RigiScan, snap indicator gauge, nocturnal electrobioimpedance volumetric assessment, sleep laboratory testing, the erectometer, the stamp test, and the mercury strain gauge. The most cost-effective method is the stamp test, but the most extensively used and trusted tool is RigiScan. ${ }^{67}$

\section{Conclusion}

Nocturnal penile tumescence are the natural erections present in all healthy men during sleep. Absence of NPT can be a good indicator for future dysfunction of reproductive system especially the ED. Lack of NPT periods can be a common characteristic of many underlying comorbid conditions. Erectile dysfunction has direct correlation with many vascular and metabolic disorders such as CVD, stroke, diabetes, obesity, and hypertension. Early diagnosis of $\mathrm{ED}$ and timely treatment of concomitant conditions can be done by asking about NPT to person during clinical diagnosis. In crux, the measurements of NPT should be contemplated as a vital diagnostic instrument and a useful marker for early detection of ED and its related comorbid conditions.

\section{References}

1. Wei M, Macera CA, Davis DR, et al. Total cholesterol and high density lipoprotein cholesterol as important predictors of erectile dysfunction. Am J Epidemiol 1994;140(10):930-937. DOI: 10.1093/ oxfordjournals.aje.a117181.

2. Giuliano FA, Leriche A, Jaudinot EO, et al. Prevalence of ED among 7689 patients with diabetes or hypertension, or both. Urology 2004;64(6):1196-1201. DOI: 10.1016/j.urology.2004.08.059.

3. Montorsi P, Ravagnani PM, Galli S, et al. The artery size hypothesis: a macrovascular link between ED and coronary artery disease. Am J Cardiol 2005;96(12B):19-23. DOI: 10.1016/j.amjcard.2005.07.006.

4. Tok A, Eminaga O, Burghaus L, et al. Age-stratified cut-off points for the nocturnal penile tumescence measurement using nocturnal electrobioimpedance volumetric assessment (NEVA $\left({ }^{\oplus}\right)$ ) in sexually active healthy men. Andrologia 2016;48(6):631-636. DOI: 10.1111/ and.12492.

5. Zou Z, Lin H, Zhang Y, et al. The role of nocturnal penile tumescence and rigidity (NPTR) monitoring in the diagnosis of psychogenic erectile dysfunction: a review. Sex Med Rev 2019;7(3):442-454. DOI: 10.1016/j.sxmr.2018.10.005. 
6. Tan HM, Tong SF, Ho CCK. Men's health: sexual dysfunction, physical, and psychological health-is there a link? J Sex Med 2012;9(3): 663-671. DOI: 10.1111/j.1743-6109.2011.02582.x.

7. World Health Organization, Defining sexual health. Report of a technical consultation on sexual health 28-31 January 2002, Geneva. WHO: Geneva; 2006. p. 5. Available at http://www.who.int/ reproductivehealth/publications/sexual_health/defining_sexual_ health.pdf (accessed January 15, 2011).

8. Feldman $H A$, Johannes $C B$, Derby $C A$, et al. ED and coronary risk factors: prospective results from the Massachusetts male aging study. Prev Med 2000;30(4):328-338. DOI: 10.1006/pmed.2000.0643.

9. El-Sakka Al. Association of risk factors and medical comorbidities with male sexual dysfunctions. J Sex Med 2007;4(6):1691-1700. DOI: 10.1111/j.1743-6109.2006.00342.x.

10. Lue TF. Erectile dysfunction. New Engl J Med 2000;342(24):1802-1813. DOI: 10.1056/NEJM200006153422407.

11. Davis JB, Tiefer L, Melman A. Accuracy of the initial history and physical examination to establish the etiology of erectile dysfunction. Urology 1995;45(3):498-502. DOI: 10.1016/S0090-4295(99)80022-3.

12. Padma-Nathan H. My approach to evaluating erectile dysfunction. Contemp Urol 1995;3:53-63.

13. Feldman HA, Goldstein I, Hatzichristou DG, et al. Impotence and its medical and psychosocial correlates: results of the Massachusetts male ageing study. J Urol 1994;151(1):54-61. DOI: 10.1016/s00225347(17)34871-1.

14. Bener A, Al-Hamaq AOAA, Kamran S, et al. Prevalence of erectile dysfunction in male stroke patients, and associated co-morbidities and risk factors. Int Urol Nephrol 2008;40(3):701-708. DOI: 10.1007/ s11255-008-9334-y.

15. Jannini EA, McCabe MP, Salonia A, et al. Organic vs. psychogenic? The Manichean diagnosis in sexual medicine. J Sex Med 2010;7(5): 1726-1733. DOI: 10.1111/j.1743-6109.2010.01824.x.

16. Huang YP, Zhang YD, Gao Y, et al. Abnormal endothelial function in ED patients with normal nocturnal penile tumescence and rigidity: is it the role of psychogenic factors? Int J Impot Res 2012;24(6):247-250. DOI: 10.1038/ijir.2012.26.

17. Hackett $G$. The burden and extent of comorbid conditions in patients with erectile dysfunction. Int J Clin Pract 2009;63(8):1205-1213. DOI: 10.1111/j.1742-1241.2009.02088.x.

18. Rosen R, Altwein J, Boyle P, et al. Lower urinary tract symptoms and male sexual dysfunction: the multinational survey of the sgeing male (MSAM-7). Eur Urol 2003;44(6):637-649. DOI: 10.1016/ j.eururo.2003.08.015.

19. Montorsi P, Montorsi F, Schulman CC. Is erectile dysfunction the "tip of the iceberg" of a systemic vascular disorder? Eur Urol 2003;44(3): 352-354. DOI: 10.1016/s0302-2838(03)00307-5.

20. Hatzimouratidis K, Amar E, Eardley I, et al. European association of urology. guidelines on male sexual dysfunction: ED and premature ejaculation. Eur Urol 2010;57(5):804-814. DOI: 10.1016/ j.eururo.2010.02.020.

21. Korpelainen JT, Nieminen P, Myllyla VV. Sexual functioning among stroke patients and their spouses. Stroke 1999;30(4):715-719. DOI: 10.1161/01.str.30.4.715.

22. Giaquinto S, Buzzelli S, Di Francesco L, et al. Evaluation of sexual changes after stroke. J Clin Psychiatry 2003;64(3):302-307. DOI: 10.4088/jcp.v64n0312.

23. Monga TN, Ostermann HJ. Sexuality and sexual adjustment in stroke patients. Phys Med Disabil Rehabil State Art Rev 1998;20:317-329.

24. Kimura M, Murata $Y$, Shimoda K, et al. Sexual dysfunction following stroke. Compr Psychiatry 2001;42(3):217-222. DOI: 10.1053/ comp.2001.23141.

25. Carod J, Egido J, Gonzalex JL, et al. Post stroke sexual dysfunction and quality of life. Stroke 1999;30(10):2238-2248. DOI: 10.1161/01. str.30.10.2238d.

26. Ponholzer A, Temml C, Obermayr R, et al. Is erectile dysfunction an indicator for increased risk of coronary heart disease and stroke? Eur Urol 2005;48(3):512-518. DOI: 10.1016/j.eururo.2005.05.014.
27. Jung JH, Kam SC, Choi SM, et al. Sexual dysfunction in male stroke patients: correlation between brain lesions and sexual function. Urology 2008;71(1):99-103. DOI: 10.1016/j.urology.2007.08.045.

28. Korpelainen JT, Kauhanen ML, Kemola $\mathrm{H}$, et al. Sexual dysfunction in stroke patients. Acta Neurol Scand 1998;98(6):400-405. DOI: 10.1111/j.1600-0404.1998.tb07321.x.

29. Böhm M, Baumhäkel M, Probstfield JL, et al. Sexual function, satisfaction, and association of ED with cardiovascular disease and risk factors in cardiovascular high-risk patients: substudy of the ongoing telmisartan alone and in combination with ramipril global endpoint trial/telmisartan randomized Assessment study in ACE-intolerant subjects with cardiovascular disease (ONTARGET/ TRANSCEND). Am Heart J 2007;154(1):94-101. DOI: 10.1016/ j.ahj.2007.03.024.

30. Solomon H, Man JW, Jackson G. Erectile dysfunction and the cardiovascular patient: endothelial dysfunction is the common denominator. Heart 2003;89(3):251-253. DOI: 10.1136/heart.89.3.251.

31. Uslu N, Gorgulu S, Alper AT, et al. Erectile dysfunction as a generalized vascular dysfunction. J Am Soc Echocardiogr 2006;19(3):341-346. DOI: 10.1016/j.echo.2005.09.017.

32. Foresta C, Caretta N, Lana A, et al. Circulating endothelial progenitorcells in subjects with erectile dysfunction. Int J Impot Res 2005;17(3):288-290. DOI: 10.1038/sj.ijir.3901311.

33. Kloner R, Padma-Nathan $\mathrm{H}$. Erectile dysfunction in patients with coronary artery disease. Int J Impot Res 2005;17(3):209-215. DOI: 10.1038/sj.ijir.3901309.

34. Kirby M. Lipid management can reduce CHD in diabetes. Best Pract 2001;26:31-32.

35. Gandaglia G, Briganti A, Jackson G, et al. A systematic review of the association between erectile dysfunction and cardiovascular disease. Eur Urol 2014;65(5):968-978. DOI: 10.1016/j.eururo.2013.08.023.

36. Corona G, Maggi M. The role of testosterone in erectile dysfunction. Nat Rev Urol 2010;7(1):46-56. DOI: 10.1038/nrurol.2009.235.

37. Kaplan SA, Meehan AG, Shah A. The age related decrease in testosterone is significantly exacerbated in obese men with the metabolic syndrome. what are the implications for the relatively high incidence of erectile dysfunction observed in these men? J Urol 2006;176(4 Pt 1):1524-1527. DOI: 10.1016/j.juro.2006.06.003.

38. Ferrannini E, Natali A, Bell P, et al. Insulin resistance and hypersecretion in obesity. European group for the study of insulin resistance (EGIR). Journal of Clinical Investigation 1997;100(5):1166-1173. DOI: 10.1172/ JCl119628.

39. Travison TG, Shabsigh R, Araujo AB, et al. The natural progression and remission of erectile dysfunction: results from the Massachusetts male aging study. J Urol 2007;177(1):241-246. DOI: 10.1016/ j.juro.2006.08.108.

40. Tan WS, Ng CJ, Khoo EM, et al. The triad of erectile dysfunction, testosterone deficiency syndrome and metabolic syndrome: findings from a multi-ethnic asian men study (the Subang men's health study). Aging Male 2011;14(4):231-236. DOI: 10.3109/13685538.2011.597463.

41. Riedner CE, Rhoden EL, Ribeiro EP, et al. Central obesity is an independent predictor of erectile dysfunction in older men. J Urol 2006;176(4 Pt 1):1519-1523. DOI: 10.1016/j.juro.2006.06.049.

42. Corona G, Mannucci E, Forti G, et al. Hypogonadism, ED, metabolic syndrome and obesity: a pathological link supporting cardiovascular diseases. Int J Androl 2009;32(6):587-598. DOI: 10.1111/j.13652605.2008.00951.x.

43. Bacon CG, Mittleman MA, Kawachi I, et al. Sexual function in men older than 50 years of age: results from the health professionals follow-up study. Ann Intern Med 2003;139(3):161-168. DOI: 10.7326/0003-4819139-3-200308050-00005.

44. Chung WS, Sohn JH, Park YY. Is obesity an underlying factor in erectile dysfunction. Eur Urol 1999;36(1):68-70. DOI: 10.1159/000019929.

45. Zumoff B, Strain GW, Miller LK, et al. Plasma free and non-sexhormonebinding-globulin-bound testosterone are decreas ED in obese men in proportion to their degree of obesity. J Clin Endocrinol Metab 1990;71(4):929-931. DOI: 10.1210/jcem-71-4-929. 
46. Vermeulen A, Kaufman JM, Deslypere JP, et al. Attenuated luteinizing hormone (LH) pulse amplitude but normal LH pulse frequency, and its relation to plasma androgens in hypogonadism of obese men. J Clin Endocrinol Metab 1993;76(5):1140-1146. DOI: 10.1210/ jcem.76.5.8496304.

47. Giagulli VA, Kaufman JM, Vermeulen A. Pathogenesis of the decreased androgen levels in obese men. J Clin Endocrinol Metab 1994;79(4):997-1000. DOI: 10.1210/jcem.79.4.7962311.

48. Derby CA, Zilber S, Brambilla D, et al. Body mass index, waist circumference and waist to hip ratio and change in sex steroid hormones: the Massachusetts male Ageing study. Clin Endocrinol (Oxf) 2006;65(1):125-131. DOI: 10.1111/j.1365-2265.2006.02560.x.

49. Kaukua J, Pekkarinen T, Sane T, et al. Sex hormones and sexual function in obese men losing weight. Obes Res 2003;11(6):689-694. DOI: 10.1038/oby.2003.98.

50. Niskanen L, Laaksonen DE, Punnonen K, et al. Changes in sex hormone-binding globulin and testosterone during weight loss and weight maintenance in abdominally obese men with the metabolic syndrome. Diabetes Obes and Metab 2004;6(3):208-215. DOI: 10.1111/j.1462-8902.2004.00335.x.

51. Isidori AM, Caprio M, Strollo F, et al. Leptin and androgens in male obesity: evidence for leptin contribution to reduced androgen levels. J Clin Endocrinol Metab 1999;84(10):3673-3680. DOI: 10.1210/ jcem.84.10.6082.

52. Isidori AM, Giannetta E, Greco EA, et al. Effects of testosterone on body composition, bone metabolism and serum lipid profile in middle-aged men: a meta-analysis. Clin Endocrinol (Oxf) 2005;63(3):280-293. DOI: 10.1111/j.1365-2265.2005.02339.x.

53. Yudkin JS, Stehouwer CD, Emeis JJ, et al. C-reactive protein in healthy subjects: associations with obesity, insulin resistance, and endothelial dysfunction: a potential role for cytokines originating from adipose tissue? Arterioscler Thromb Vasc Biol 1999;19(4):972-978. DOI: 10.1161/01.atv.19.4.972.

54. Diaz-Arjonilla M, Schwarcz M, Swerdloff RS, et al. Obesity, low testosterone levels and erectile dysfunction. Int J Impot Res 2009;21(2):89-98. DOI: 10.1038/ijir.2008.42.

55. Yassin AA, Saad F, Gooren LJ. Metabolic syndrome, testosterone deficiency and ED never come alone. Andrologia 2008;40(4):259-264. DOI: 10.1111/j.1439-0272.2008.00851.x.
56. Hackett GI. Impotence - the most neglect erectile dysfunction complication of diabetes. Diabetes Res 1995;28:75-83.

57. Eardley I, Fisher W, Rosen RC, et al. The multinational men's attitudes to life events and sexuality study: the influence of diabetes on selfreported erectile function, attitudes and treatment-seeking patterns in men with erectile dysfunction. Int J Clin Pract 2007;61(9):1446-1453. DOI: 10.1111/j.1742-1241.2007.01460.x.

58. Burchardt M, Burchardt T, Baer L, et al. Hypertension is associated with severe erectile dysfunction. J Urol 2000;164(4):1188-1191. DOI: 10.1016/S0022-5347(05)67138-8.

59. Dusing R. Effect of the angiotensin II antagonist valsartan on sexual function in hypertensive men. Blood Press Suppl 2003;2(sup2):29-34. DOI: 10.1080/08038020310021967.

60. Seftel AD, Sun P, Swindle R. The prevalence of hypertension, hyperlipidemia, diabetes mellitus and depression in men with erectile dysfunction. J Urol 2004;171(6 Pt 1):2341-2345. DOI: 10.1097/01. ju.0000125198.32936.38.

61. Karacan I. Clinical value of nocturnal erection in the prognosis and diagnosis of impotence. Med Aspects of Human Sexuality 1970;4:2734.

62. Fisher C, Gross J, Zuch J. Cycle of penile erection synchronous with dreaming (REM) sleep. Arch Gen Psychiatry 1965;12(1):29-45. DOI: 10.1001/archpsyc.1965.01720310031005.

63. Moreland RB. Is there a role of hypoxemia in penile fibrosis: a viewpoint presented to the society for the study of Impotence. Int J Impot Res 1998;10(2):113-120. DOI: 10.1038/sj.ijir.3900328.

64. Yilmaz E, Yaman O, Bozlu M, et al. Comparison of nocturnal penile tumescence monitoring and cavernosal smooth muscle content in patients with erectile dysfunction. Int Urol Nephrol 2002;34(1): 117-120. DOI: 10.1023/a:1021327500439.

65. Levine LA, Carroll RA. Nocturnal penile tumescence and rigidity in men without complaints of ED using a new quantitative analysis software. J Urol 1994;152(4):1103-1107. DOI: 10.1016/s00225347(17)32514-4.

66. Halverson HM.Genitaland sphincter behavior of the maleinfant.JGenet Psychol 1940;56(1):95-136. DOI: 10.1080/08856559.1940.9944066.

67. Qin F, Gao L, Qian S, et al. Advantages and limitations of sleeprelated erection and rigidity monitoring: a review. Int J Impot Res 2018;30(4):192-201. DOI: 10.1038/s41443-018-0032-8. 